\title{
Relationships of self-reported physical activity, fitness and body mass index with inflammatory proteins in adolescents. The AFINOS study
}

\author{
D. Martínez-Gómez ${ }^{1,2}$, J. C. Eisenmann ${ }^{3}, \mathrm{~S}_{\text {. Gómez-Martínez }}{ }^{1}$, J. Wärnberg ${ }^{1}$, A. M. Veses ${ }^{1}$, \\ O. L. Veiga ${ }^{2}$ and A. Marcos ${ }^{1}$ \\ ${ }^{1}$ Immunonutrition Research Group, Department of Metabolism and Nutrition, Instituto del Frio-ICTAN, Spanish National \\ Research Council (CSIC), Madrid, Spain, ${ }^{2}$ Department of Physical Education, Sport and Human Movement, Facultad \\ de Formación del Profesorado y Educación, Universidad Autonoma de Madrid, Madrid, Spain and ${ }^{3}$ Departments of \\ Kinesiology and Pediatrics \& Human Development, East Lansing, MI, USA
}

Inflammatory proteins during childhood and adolescence have been shown to be predictors of cardiovascular disease in adulthood ${ }^{(1)}$. Likewise, higher levels of body fat seem to play a pro-inflammatory role in adolescents ${ }^{(2)}$. However, there is limited knowledge about the role of physical activity and fitness on inflammatory proteins in adolescents. Therefore, the aim of this study was to examine the relationships between self-reported physical activity (PA), fitness, body mass index (BMI), and inflammatory proteins. A sub-sample of 193 adolescents (92 girls), aged 13-17 years old and enrolled in the AFINOS study, was selected for the current study. PA was assessed by a global activity rating (compared to others of your age and sex, how much PA do you get?). Similarly, fitness was self-reported by the following question: how is your physical fitness? Both PA and fitness assessments were rated on a 5-point scale ranging from 1 (much less active/very bad) to 5 (much more active/excellent). Weight and height were also obtained by self-report and BMI was calculated $\left(\mathrm{kg} / \mathrm{m}^{2}\right)$. C-reactive protein $(\mathrm{CRP}, \mathrm{mg} / \mathrm{dl})$, ceruloplasmin $(\mathrm{mg} / \mathrm{dl})$ and complement factors $\mathrm{C} 3$ and $\mathrm{C} 4(\mathrm{~g} / \mathrm{l})$ were determined, and logarithmic or square roots transformations were applied when necessary. Student's $t$ test showed no differences between sexes regarding age and BMI. Adolescent boys were more active and fit than girls (both $P=0.001$ ). No sex differences were found for inflammatory proteins, except for ceruloplasmin, adolescent girls reaching higher levels than boys $(P=0.002)$. PA was significantly related with fitness $(r=0.546$, $P<0.001)$ but not with BMI $(r=-0.132, P=0.077)$ after controlling for age and sex. Fitness was also related with BMI $(r=-0.301$, $P<0.001)$ after controlling for age and sex.

Table 1. Partial correlations controlling for age and sex $(n=193)$

\begin{tabular}{lcccr}
\hline & $\operatorname{Ln}(\mathrm{CRP})$ & Sqrt(Ceruloplasmin) & Sqrt(C3) & Sqrt(C4) \\
\hline PA (score) & -0.046 & -0.133 & -0.131 & -0.110 \\
Fitness $(\mathrm{score})$ & $-0.220^{*}$ & $-0.257^{*}$ & $-0.341^{*}$ & $-0.270^{*}$ \\
BMI $\left(\mathrm{kg} / \mathrm{m}^{2}\right)$ & $0.307^{*}$ & $0.214^{*}$ & $0.454^{*}$ & $0.336^{*}$ \\
\hline
\end{tabular}

$* P<0.05$.

PA was not significantly related/associated with inflammatory proteins, but borderline significances were found with ceruloplasmin $(P=0.066)$ and $\mathrm{C} 3(P=0.072)$. On the contrary, fitness and BMI were significantly related with all the inflammatory proteins tested. When BMI was included as confounder, fitness was only significantly related with celluloplasmin $(r=-0.195, P=0.008)$ and C3 ( $r=-0.216, P=0.003)$. These results suggest that PA has a potential beneficial effect on low-grade inflammation in adolescents due to its role on fitness.

1. Juonala M, Viikari JS, Rönnemaa T et al. (2006) Arterioscler Thromb Vasc Biol 26, 1883-1888.

2. Wärnberg J, Nova E, Moreno LA et al. (2006) Am J Clin Nutr 84, 505-512. 